\title{
Carrier Detection in Families with Properdin Deficiency by Microsatellite Haplotyping
}

Konrad Kölble, * Andrew J. Cant, ${ }^{*}$ Anne C. Fay, ${ }^{5}$ Keith Whaley," Menachem Schlesinger," and Kenneth B. M. Reid * ${ }^{*}$ Medical Research Council Immunochemistry Unit, Department of Biochemistry, University of Oxford, Oxford OX1 $3 Q U$; ${ }^{\ddagger}$ Children's Department and ${ }^{\S}$ Pathology Laboratory, Newcastle General Hospital, Newcastle NE4 6BE; "Department of Immunology, Leicester Royal Infirmary, Leicester LE1 5WW, United Kingdom; and 'Department of Immunology, Barzilai Medical Center, Ashkelon, 78306, Israel

\begin{abstract}
Human properdin deficiency is an X-linked disorder strongly predisposing to meningococcal disease which has been recorded in over 50 cases of various ethnic origins. Immunochemically, total deficiency (type I), partial deficiency (type II), and deficiency due to a dysfunctional molecule (type III) can be differentiated. It is therefore most likely that the causative molecular defects will show considerable genetic heterogeneity. Analysis of the properdin locus at Xp11.3-Xp11.23 has led to the characterization of two polymorphic $(\mathrm{dC}-\mathrm{dA})_{\mathrm{n}} \cdot(\mathrm{dG}-\mathrm{dT})_{\mathrm{n}}$ repeats located $\sim 15 \mathrm{~kb}$ downstream from the structural gene. Three families (two Scottish Caucasoid, one Tunisian Sephardic) with seven deficient individuals were investigated immunochemically and using a nonradioisotopic polymerase chain reaction-based method for microsatellite detection. Probable and definite carriers frequently showed properdin levels which were in the normal range. No recombinants between the microsatellite loci and properdin deficiency were detected, thus allowing identification of the defective allele through the generations in all three pedigrees. Haplotyping for these highly polymorphic microsatellites in close physical linkage to the properdin gene can provide rapid and nonradioactive detection of carrier status and prenatal diagnosis without extensive sequencing analysis. (J. Clin. Invest. 1993. 91:99-102.) Key words: polymorphism genetics • repetitive sequences $\bullet$ genetic markers genetics • complement pathway, alternative immunology • polymerase chain reaction
\end{abstract}

\section{Introduction}

Properdin stabilizes the alternative $\mathrm{C} 3$ convertase $(\mathrm{C} 3 \mathrm{~b}, \mathrm{Bb})$ of the complement system and thereby enhances the efficiency of the alternative pathway (1). Human properdin deficiency is an $\mathrm{X}$-linked disorder that strongly predisposes to meningococcal disease. In contrast to deficiencies of the late complement components, i.e., $\mathrm{C} 6, \mathrm{C} 7$, and $\mathrm{C} 8$, properdin deficiency is not usually associated with recurrent disease but shows a higher

This work has appeared in abstract form (1991. Complement Inflammation. 8:176).

Address correspondence to Konrad Kölble, M. D., Ph.D., Medical Research Council Immunochemistry Unit, Department of Biochemistry, University of Oxford, South Parks Road, Oxford, OX1 3QU, United Kingdom. 1992.

Received for publication 1 June 1992 and in revised form 27 July

J. Clin. Invest.

(C) The American Society for Clinical Investigation, Inc.

0021-9738/93/01/99/04 \$2.00

Volume 91, January 1993, 99-102 case / fatality rate. While the exact mechanisms for the predominance of Gram-negative infections in the properdin-deficient individuals and the apparent absence of infectious complications in some of the affected remain to be elucidated, the more than 50 cases described to date demonstrate the need for diagnostic techniques to allow prenatal and carrier detection $(2,3)$. Three different types of properdin deficiency have been described on the basis of immunochemical and functional analyses (2). Type I is characterized by protein levels below the detection limit of standard immunochemical methods (RIA or ELISA) and undetectable properdin function. In type II properdin serum levels are reduced to levels between $\sim 1$ and $10 \%$ of normal $(4,5)$, the inclusion of partial deficiency cases with concentrations between 20 and $35 \%$ of normal in this category remains questionable as $\mathrm{X}$-linked transmission has not been clearly demonstrated $(6,7)$. Finally, in type III properdin deficiency normal amounts of properdin are antigenically detectable but the protein appears functionally defective ( 8$)$. This phenotypic heterogeneity together with the variable ethnic and geographic origin of the patients suggest that the disorder is most likely genetically heterogeneous with different mutations present in the various pedigrees.

Recently the cloning and sequencing of the human properdin locus at Xp1 1.3-Xp1 1.23 has led to the characterization of a dinucleotide repeat containing sequence $<15 \mathrm{~kb}$ downstream of the properdin structural gene $(9,10)$. This region encompasses two perfect $(\mathrm{dC}-\mathrm{dA})_{\mathrm{n}} \cdot(\mathrm{dG}-\mathrm{dT})_{\mathrm{n}}$ repeats interrupted by $83 \mathrm{bp}$. Here we report the use of haplotyping of these two microsatellite markers for the carrier detection of properdin deficiency.

\section{Methods}

Subjects. A total of 27 individuals from three families (Tunisian Sephardic family 1 , Scottish Caucasoid families 2 and 3 ) including 7 properdin-deficient individuals, 4 obligate carriers, and 5 potential carriers were studied on the DNA level (Fig. 1). Families 1 and 2 have been reported previously $(11,12)$.

The index case of family 3 presented with acute epiglottitis at the age of $3 \mathrm{yr}$ which responded to antibiotics. At the age of $9 \mathrm{yr}$ he developed septic meningitis with a purpuric rash. Neisseria meningitidis was grown from blood and cerebrospinal fluid cultures. Treatment with penicillin and assisted ventilation resulted in a good recovery though occasional left-side seizures were observed. Investigations for possible immunodeficiencies in this patient and his younger brother who had mild umbilical sepsis as a neonate and an episode of pneumonia at $4 \mathrm{yr}$ showed normal immunoglobulin and immunoglobulin subclass levels but markedly diminished alternative pathway function. There was no family history of sepsis, meningitis, or recurrent infections apart from a maternal uncle's death of pneumonia at the age of 30 .

Functional and protein assays. Hemolytic activity of the alternative pathway of complement (AP50) was measured by the lysis of rabbit erythrocytes in buffers containing $2.5 \mathrm{mM} \mathrm{MgCl}_{2}$ and $10 \mathrm{mM}$ EGTA 

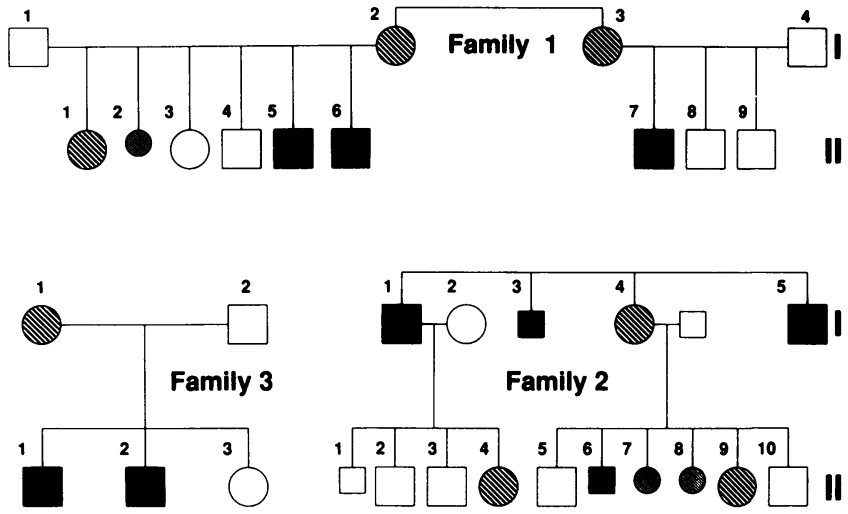

Figure 1. Pedigrees of the three families with properdin deficiency. Solid squares identify deficient individuals, hatched circles denote presence of the deficiency-linked microsatellite haplotype $(00$ in family 3, 02 in families 1 and 2), i.e., genotypic carrier status. The smaller symbols indicate that DNA was not available for study. The solid squares indicate immunochemically deficient individuals, the smaller hatched circles indicate potential carrier status.

(13). The following sandwich enzyme-linked immunosorbent assay was used to measure the concentration of properdin in plasma and serum samples: wells were coated with the IgG fraction of a polyclonal rabbit anti-human properdin antiserum prepared and purified using standard techniques ( $150 \mu \mathrm{l}$ of $1 / 2,000$ dilution of $15 \mathrm{mg} / \mathrm{ml}$ solution ); samples $(150 \mu \mathrm{l})$ of plasma, serum, or purified properdin were added and incubated at $37^{\circ} \mathrm{C}$ for $2 \mathrm{~h}$; the bound properdin was detected by the addition of the biotinylated IgG fraction from rabbit anti-human properdin ( $150 \mu \mathrm{l}$ of $1 / 2,000$ dilution of $10 \mathrm{mg} / \mathrm{ml}$ solution ), streptavidinalkaline phosphatase ( $150 \mu \mathrm{l}$ of $1 / 10,000$ dilution) and phosphatase substrate $(50 \mu \mathrm{l}$ of $1 \mathrm{mg} / \mathrm{ml}$ solution of $p$-nitrophenyl disodium phosphate). The plates were monitored at $405 \mathrm{~nm}$ after $15 \mathrm{~min}$. All dilutions were made in Tris-buffered saline $\mathrm{pH} 7.4$ containing $0.5 \mathrm{mg} / \mathrm{ml}$ BSA. The assay was sensitive down to a level of $1 \mathrm{ng} / \mathrm{ml}$ of human properdin.

Microsatellite typing. DNA of properdin-deficient individuals, their families, and the control population were prepared either as fluid phase DNA according to standard protocols or as agarose-embedded

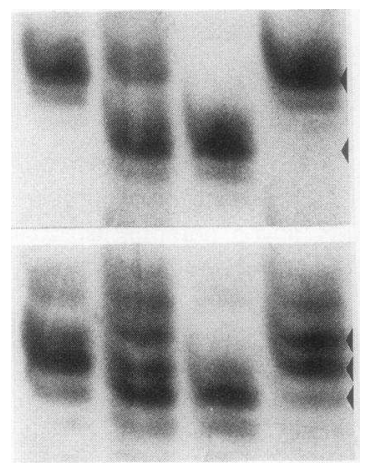

Figure 2. Electrophoresis of doublestranded polymerase chain reaction products on a nondenaturing polyacrylamide gel and subsequent photochemical silver staining. Amplification across dinucleotide repeats typically produces a multi-banded pattern consisting of a major band and additional "shadow" bands migrating slower, i.e., as a cathodal minor band, and faster, i.e., as an anodal minor band. The molecular basis for this phenomenon is not fully understood: strand slippage or staggered pairing of partially extended products during early cycles are possible explanations. To assist in allele identification major band levels are marked by arrowheads on both panels. The upper panel depicts the region of the gel containing the repeat 1 patterns; the lower panel depicts the region of the gel containing the repeat 2 patterns. Lanes (from left to right) are: $(A)$ Family 3 I-2 Repeat 1 phenotype 6 , Repeat 2 phenotype $2 ;(B)$ Family 3 I-1 Repeat 1 phenotype 0/6, Repeat 2 phenotype $0 / 4 ;(C)$ Family 3 II-2 Repeat 1 phenotype 0 , Repeat 2 phenotype $0 ;(D)$ Family 3 II-3 Repeat 1 phenotype 6 , Repeat 2 phenotype $2 / 4$.
Table I. Haplotype Frequencies for the Properdin Microsatellites Derived from Typing 119 X Chromosomes of CEPH Parents

\begin{tabular}{|c|c|c|c|}
\hline \multicolumn{2}{|c|}{ Haplotype } & \multirow{2}{*}{$\begin{array}{l}\text { Number } \\
n=119\end{array}$} & \multirow[b]{2}{*}{ Frequency } \\
\hline Repeat 1 & Repeat 2 & & \\
\hline 0 & 0 & 43 & 0.361 \\
\hline 6 & 4 & 25 & 0.210 \\
\hline 0 & 2 & 13 & 0.109 \\
\hline 8 & 4 & 10 & 0.084 \\
\hline 12 & 2 & 6 & 0.050 \\
\hline 0 & 4 & 4 & 0.034 \\
\hline 2 & 0 & 4 & 0.034 \\
\hline-2 & 0 & 3 & 0.025 \\
\hline 4 & 0 & 3 & 0.025 \\
\hline 0 & -2 & 2 & 0.017 \\
\hline 10 & 4 & 2 & 0.017 \\
\hline 12 & 4 & 2 & 0.017 \\
\hline-2 & 4 & 1 & 0.008 \\
\hline 6 & 6 & 1 & 0.008 \\
\hline
\end{tabular}

Reference level 0 designates the most frequent allele.

chromosomal DNA (14). Polymerase chain reactions were carried out in $50-\mu \mathrm{l}$ vol overlaid with $20 \mu \mathrm{l}$ liquid paraffin. The reaction mix contained $\sim 100 \mathrm{ng}$ genomic DNA template, $1 \mu \mathrm{M}$ of each oligonucleotide primer (repeat 1: PCA5 5' CCTGAGGATAGTGTCAGCGAT 3' [GT strand; nucleotides 1-21 of GenBank M64474 sequence] [10]) and PCA43 5' GCACAAATGGACCCTGGTGACT 3' (CA strand; nucleotides 138-117 of GenBank M64474 sequence); repeat 2: PCA45 5' AGTCACCAGGGTCCATTTGTGC 3' (GT strand; nucleotides 117-138 of GenBank M64474 sequence), PCA3 5' CTTTCAGGGCTACTGGTCACT 3' (CA strand; nucleotides 224-204 of GenBank M64474 sequence) and $2.5 \mathrm{mM} \mathrm{MgCl}, 10 \mathrm{mM}$ TRIS (pH 8.3), 50 $\mathrm{mM} \mathrm{KCl}, 0.01 \%$ gelatin, $1 \mathrm{mM}$ of each dNTPs, and $20 \mathrm{mU} / \mu \mathrm{l}$ Taqpolymerase used 30 cycles at $94^{\circ} \mathrm{C}, 60^{\circ} \mathrm{C}$, and $72^{\circ} \mathrm{C}$ for $30 \mathrm{~s}$ each. A 1:20 of each reaction was mixed with 0.2 vol of glycerol dyes and run on $40 \mathrm{~cm}$ long $0.4 \mathrm{~mm}$ thick, nondenaturing, $5.5 \%$ (wt/vol) acrylamide gels at $40 \mathrm{~W}$ under constant power mode for $2 \mathrm{~h}$. DNA was visualized using a photochemical silver stain (Bio-Rad Laboratories, Richmond, CA). Sizes of the alleles were determined by comparison to size markers (Gibco BRL, Bethesda, MD).

\section{Results}

Microsatellite haplotyping. The region containing the two microsatellites has initially been amplified en bloc using the primers PCA5 and PCA3 (10). This combined approach, however, does not differentiate between the various reciprocal alterations in dinucleotide repeat number at the individual loci that would result in identically sized amplification products. Therefore additional primers were selected to allow the individual amplification of each repeat (PCA5 and PCA43 for repeat 1 and PCA45 and PCA3 for repeat 2). The sizes of the amplified products vary in steps of two bp above and below, the 138-bp allele 0 for repeat 1 and the 108 -bp allele 0 for repeat 2 . This permits simultaneous separation of both sets of reaction products in the same lane of a nondenaturing gel (Fig. 2). Polyacrylamide electrophoresis and silver staining of the doublestranded polymerase chain reaction products yielded clear banding patterns consisting typically of two major bands and an anodal minor band in homo- and hemizygotes. Following 
Table II. Microsatellite Genotypes in Members of Three Families with Properdin Deficiency

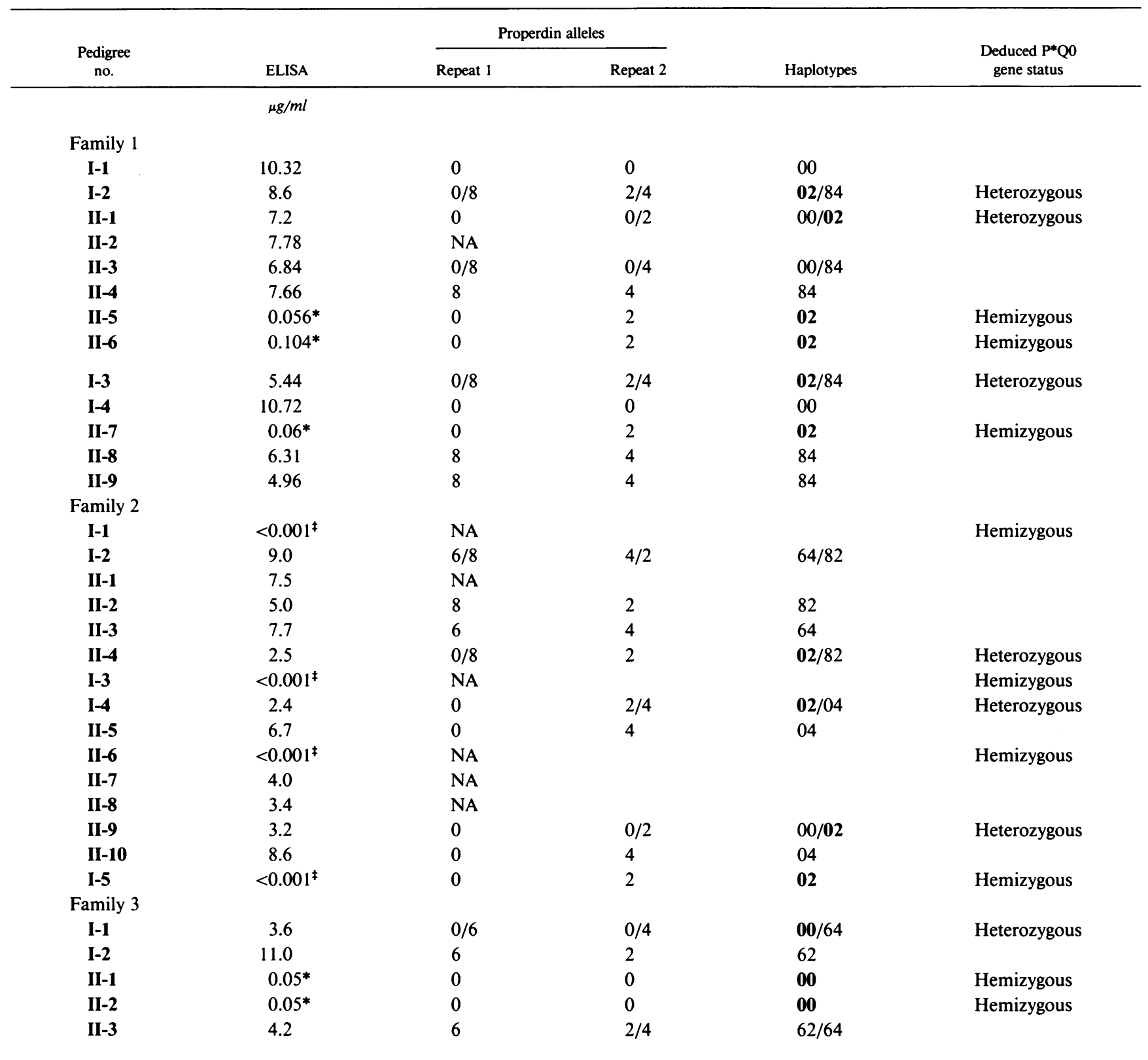

Haplotypes carrying the defective allele are in bold face. NA indicates DNA samples not available. * Type II deficiency; ${ }^{*}$ type I deficiency.

an earlier suggestion the size of the most frequent allele was taken as the reference point (the zero level) and the other alleles designated in relation to this zero level (15). In the present sample, eight alleles have been identified for repeat 1 and five alleles have been identified for repeat 2 . Alleles at both loci can be combined in a haplotype thus yielding a highly informative marker system. In $119 \mathrm{X}$-chromosomes of Centre d'Etude du Polymorphisme Humaine $(\mathrm{CEPH})^{1}$ parents 14 haplotypes were observed and 5 of these occurred at a frequency $5 \%$ or higher ( Table I). Haplotype heterozygosity in 39 CEPH family mothers was $>87 \%$.

1. Abbreviation used in this paper: CEPH, Centre d'Etude du Polymorphisme Humaine.
Immunochemical investigations. The serum properdin concentrations of the members of the three families were measured, and showed phenotypic heterogeneity with respect to properdin deficiency. While in the deficient individuals of family 2 (I-1, I-3, I-5, and II-6) the properdin concentration was below the detection limit of the immunosorbent assay of 1 $\mathrm{ng} / \mathrm{ml}$ the deficient individuals of family 3 (II-1 and II-2) had $\sim 0.5 \%$ of the normal serum properdin concentration $(\sim 10$ $\mu \mathrm{g} / \mathrm{ml}(16)$ ) in an ELISA (Table II). The AP50 values for plasma samples from the index case and his brother, in family 3 , were $<2 \%$ of normal levels. Reconstituting these two plasma samples to the normal level with purified human properdin raised the AP50 values to the normal range. The AP50 values for plasma samples from the father, the mother (an obligate carrier of the deficiency allele), and the daughter, in family 3 
were very similar to each other and within the normal AP50 titration range. Probable and definite carriers frequently show properdin levels and AP50 activities within the normal range. It is therefore apparent that immunochemical and functional assays do not allow the reliable differentiation between obligate and possible carriers in properdin deficiency.

Haplotyping in the properdin-deficient families. The power of the haplotype approach is demonstrated by the observed heterozygosity in all female family members in the sample. There was complete correspondence between the functional and immunological identification of deficient individuals ( Table II). The silent allele was found linked to the 02 haplotype in families 1 and 2 and to the 00 haplotype in family 3 .

\section{Discussion}

Immunochemical and functional investigations of the properdin levels in families with cases of properdin deficiency do not allow the unambiguous identification of carriers or a prenatal diagnosis of the condition (17). In all three families with properdin deficiency haplotyping for microsatellites closely linked to the properdin gene on the $\mathrm{X}$ chromosome allowed identification of the defective allele through two generations. Recombinatorial events were not observed and are, given the size of the properdin gene and the close proximity of the dinucleotide repeats, extremely unlikely. However, although the degree of heterozygosity in females for the microsatellite haplotypes described is very high compared to conventional restriction fragment length polymorphism markers and the average single microsatellite homozygosity in carriers is possible and could necessitate typing for additional flanking markers, such as the polymorphic DXS228 microsatellites (10).

Interestingly, the mutation leading to the type II deficiency in family 3 has occurred in the context of the haplotype most frequently found in 116 parental CEPH chromosomes, whereas the third most frequent haplotype carried a type II deficiency mutation in family 1 and a type I deficiency mutation in family 2 . This may indicate an increased mutability of the properdin locus characterized by the 02 haplotype but more importantly it supports the concept of independent mutational events in the three pedigrees studied and quite possibly in most of the properdin deficiencies described. Considering the phenotypic spectrum of properdin deficiency incuding dysfunctional variants (8) and various degrees of partial defects (4-7) the underlying molecular defects can be expected to be quite diverse. Such genetic heterogeneity has been directly demonstrated for $\mathrm{C} 3$ deficiency, which is also found in more than one ethnic background $(18,19)$. Comprehensive sequencing of even average size genes for small deletions, or single nucleotide changes, to uncover private mutations is not practicable for routine carrier detection. While traditional restriction fragment length polymorphism analyses can often offer only limited informativeness, the state-of-the-art screening techniques, such as single strand conformation polymorphisms, can be very laborious. Highly polymorphic microsatellite markers will therefore become increasingly popular for linkage and allelic association studies. In the case of properdin deficiency the availability of two polymorphic microsatellites closely linked to the structural locus allowed to establish microsatellite haplotypes thereby further enhancing the power of simple sequence repeat analyses. The combination of the polymerase chain reaction with a nonisotopic detection method makes the described diagnostic procedure immediately applicable in routine laboratories.

\section{Acknowledgments}

This research was supported by a grant from the National Meningitis Trust.

\section{References}

1. Pangburn, M. K., and H. J. Müller-Eberhard. 1984. The alternative pathway of complement. Springer Semin. Immunopathol. 7:163-192.

2. Sjöholm, A. G. 1990. Inherited complement deficiency states: implications for immunity and immunological disease. APMIS (Acta Pathol. Microbiol. Immunol. Scand.). 98:861-874.

3. Figueroa, J. E., and P. Densen. 1991. Infectious diseases associated with complement deficiencies. Clin. Microbiol. Rev. 4:359-395.

4. Nieisen, H. E., and C. Koch. 1987. Congenital properdin deficiency and meningococcal infection. Clin. Immunol. Immunopathol. 44:134-139.

5. Sjöholm, A. G., C. Söderström, and L. A. Nilsson. 1988. A second variant of properdin deficiency: the detection of properdin at low levels in affected males. Complement. 5:130-140.

6. Davis, C. A., and J. Forristal. 1980. Partial properdin deficiency. J. Lab. Clin. Med. 96:633-639.

7. Wyatt, R. J., B. A. Julian, and J. H. Galla. 1981. Properdin deficiency with IgA nephropathy. N. Engl. J. Med. 305:1097.

8. Sjöholm, A. G., E. J. Kuijper, C. C. Tijssen, A. Jansz, P. Bol, L. Spanjaard, and H. C. Zanen. 1988. Dysfunctional properdin in a Dutch family with meningococcal disease. N. Engl. J. Med. 319:33-37.

9. Nolan, K. F., S. Kaluz, J. M. G. Higgins, D. Goundis, and K. B. M. Reid. 1992. Characterization of the human properdin gene. Biochem. J. 287:291-297.

10. Coleman, M. P., J. C. Murray, H. F. Willard, K. F. Nolan, K. B. M. Reid, D. J. Blake, S. Lindsay, S. S. Bhattacharya, A. Wright, and K. E. Davies. 1991. Genetic and physical mapping around the properdin P gene. Genomics. 11:991996.

11. Schlesinger, M., Z. Nave, Y. Levy, P. E. Slater, and Z. Fishelson. 1990. Prevalence of hereditary properdin, $\mathrm{C} 7$ and $\mathrm{C} 8$ deficiencies in patients with meningococcal infections. Clin. Exp. Immunol. 81:423-427.

12. Holme, E. R., J. Veitch, A. Johnston, G. Hauptmann, B. Uring-Lambert, M. Seywright, V. Docherty, and K. Whaley. 1989. Familial properdin deficiency associated with chronic discoid lupus erythematosus, Clin. Exp. Immunol. 76:76-81.

13. Platts-Mills, T. A. E., and K. Ishizaka. 1974. Activation of the alternative pathway of human complement by rabbit cells. J. Immunol. 113:348-358.

14. Kölble, K., and R. B. Sim. 1991. An angle-variable three dimensional pulsed field electrophoresis system. Anal. Biochem. 191:32-38.

15. Weber, J. L., and P. E. May. 1989. Abundant class of human DNA polymorphisms which can be typed using the polymerase chain reaction. Am. J. Hum. Genet. 44:388-396.

16. Pangburn, M. K. 1989. Analysis of natural polymeric forms of human properdin and their functions in complement activation. J. Immunol. 142:202207.

17. Densen, P., J. M. Weiler, J. McLeod Griffiss, and L. G. Hoffmann. 1987. Familial properdin deficiency and fatal meningococcemia. Correction of the bactericidal defect by vaccination. $N$. Engl. J. Med. 316:922-926.

18. Botto, M., K. Y. Fong, A. K. So, A. Rudge, and M. J. Walport. 1990. Molecular basis of hereditary C3 deficiency. J. Clin. Invest. 86:1158-1166.

19. Colten, H. R., and F. S. Rosen. 1992. Complement deficiencies. Annu. Rev. Immunol. 10:809-834. 